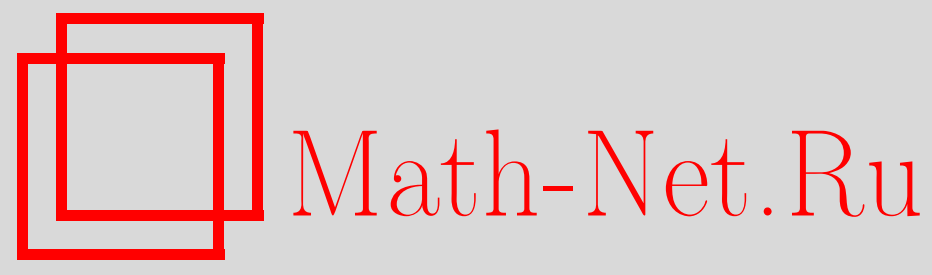

А. Н. Дегтев, Почти полурекурсивные множества, Матем. заметки, 1999, том 66, выпуск 2, 188-193

DOI: https://doi.org/10.4213/mzm1155

Использование Общероссийского математического портала Math-Net.Ru подразумевает, что вы прочитали и согласны с пользовательским соглашением http://www.mathnet.ru/rus/agreement

Параметры загрузки:

IP : 44.207 .124 .84

26 апреля 2023 г., 08:34:23

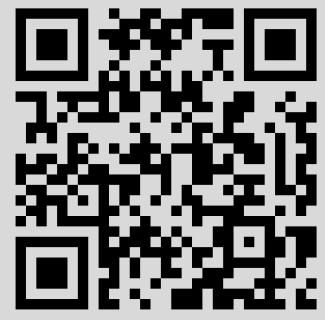


УДК 510.5

\title{
ПОЧТИ ПОЛУРЕКУРСИВНЫЕ МНОЖЕСТВА
}

\author{
А.Н. Дёгтев
}

Пусть $\mathbb{N}=\{0,1,2, \ldots\}, A \subseteq \mathbb{N}$ и $a \notin A$. Назовем $A$ почти полурекурсивным множеством, если есть двухместная общерекурсивная функция $f$ такая, что для всех $x, y \in \mathbb{N} f(x, y) \in\{x, y, a\} \wedge(\{x, y\} \subseteq A \Longleftrightarrow f(x, y) \in A)$. Доказано, в частности, что если $A$ и $\mathbb{N} \backslash A$ - почти полурекурсивные множества, то $A$ - полурекурсивное множество; что существует wsr*-множество, не являющееся ни wsr-, ни почти полурекурсивньм, и другие факты.

Библиограффия: 5 названий.

Пусть $\mathbb{N}=\{0,1,2, \ldots\}, A \subseteq \mathbb{N}$ и $\bar{a} \notin A$. Назовем $A$ почти полурекурсивны.м множеством, если найдется двухместная общерекурсивная функция $f$ такая, что для всех $x, y \in \mathbb{N}$

$$
f(x, y) \in\{x, y, \bar{a}\} \wedge(x \in A \wedge y \in A \Longleftrightarrow f(x, y) \in A) .
$$

Если в (1) заменим $\{x, y, \bar{a}\}$ на $\{x, y\}$, то придем к одному из возможных определений полурекурсивного множества [1], играющего заметную роль в теории рекурсии. Цель данной заметки - выяснить некоторые свойства таких множеств и сравнить их класс с другими классами множеств, близких к полурекурсивным.

Множество $A \subseteq \mathbb{N}$ называется слабо полурекурсивным (wsr-) [2], если найдется двухместная частично-рекурсивная функция $f$ такая, что для всех $x, y \in \mathbb{N}$

$$
((x \in A \wedge y \notin A) \vee(x \notin A \wedge y \in A)) \Longrightarrow(f(x, y) \downarrow \wedge f(x, y) \in\{x, y\} \cap A)
$$

- полурекурсивно-перечислимым (sre-), если

$$
(x \in A \vee y \in A) \Longrightarrow(f(x, y) \downarrow \wedge f(x, y) \in\{x, y\} \cap A),
$$

и шsr*-множсеством [3], если

a) $f(x, y) \downarrow \Longrightarrow f(x, y) \in\{x, y\}$;

б) $x \in A \wedge y \in A \Longrightarrow f(x, y) \downarrow$;

в) $f(x, y) \downarrow \wedge f(x, y) \in A \Longrightarrow x \in A \wedge y \in A$.

Заметим, что ранее [4] wsr*-множества были тоже названы слабо полурекурсивными.

Ясно, что каждое полурекурсивное множество является sre-множеством, а последние - wsr-множествами. Легко показать также [2], что множество $A$ полурекурсивно, если и только если $\bar{A}=\mathbb{N} \backslash A$ и $A$ есть sre-множества, a каждое рекурсивно-перечислимое множество является sre-множеством. Отсюда следует, что если 
$A$ - рекурсивно-перечислимое неполурекурсивное множество, то $\bar{A}$ является wsr-, но не wsr*-множеством [2]. С другой стороны, в [3] приведен пример wsr*-, но не wsr-множества и показано, что $A$ является sre-множеством тогда и только тогда, когда $A$ - одновременно wsr- и wsr*-множество. Что касается почти полурекурсивных множеств, то они являются wsr*-множествами.

ПРЕДЛОЖЕНИЕ 1. Каждая нерекурсивная $\mathrm{tt}-$ степень содержит почти полурекурсивное мнохсество, не являющееся слабо полурекурсивным.

ДокАЗАТЕЛЬСТво. УПорядочим множество $\Omega$ всех конечных последовательностей из 0 и 1 лексикографически:

$$
0<1<00<01<10<11<000<010<011<100<\cdots
$$

а затем занумеруем их числами $0,1,2, \ldots$ в порядке возрастания. Таким образом, определено эффективное взаимно однозначное соответствие между элементами из $\Omega$ их номерами.

Пусть

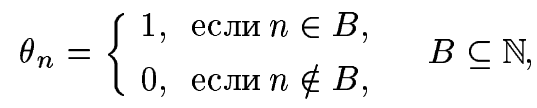

и $A=\left\{\theta_{0}, \theta_{0} \theta_{1}, \theta_{0} \theta_{1} \theta_{2}, \ldots\right\}$. Ясно, что $A={ }_{\text {tt }} B$. Будем писать $\alpha \triangleleft \beta$, если $\alpha-$ начало последовательности $\beta$. Пусть

$$
g(\alpha, \beta)= \begin{cases}\alpha, & \text { если } \beta \triangleleft \alpha, \\ \beta, & \text { если } \alpha \triangleleft \beta, \\ \gamma & \text { в противном случае, }\end{cases}
$$

где $\gamma$ - фиксированный элемент из $\Omega \backslash A$. Легко понять, что $A$ посредством общерекурсивной функции $g$ оказывается почти полурекурсивньм множеством. Предположим, что $A$ - нерекурсивное множество и для $A$ нашлась частично-рекурсивная функция $f$, удовлетворяющая (2). Но тогда если $\alpha \in A$, то

$$
f(\alpha 0, \alpha 1) \downarrow \wedge(\alpha 1 \in A \Longleftrightarrow f(\alpha 0, \alpha 1)=\alpha 1) .
$$

Это противоречит нерекурсивности множества $A$.

ТЕорема 1. Существует $\mathrm{wsr}^{*}$-множество, не являющееся ни wsr-, ни почти полурекурсивным.

ДокАЗАТЕЛЬСТво. Пусть $B$ - гиперпростое множество с ретрассируемым дополнением. Для $B$ найдется представляющая общерекурсивная функция $h$ такая, что [5]

$$
x \in B \Longleftrightarrow(\exists y)(y>x \wedge h(y) \leqslant h(x))
$$

Проверим, что $A$ является wsr*-множеством, где $A=\{2 x: x \in B\} \cup\{2 x+1: x \in \bar{B}\}$. Пусть $x, y$ - два четных числа, $x=2 x_{1}, y=2 y_{1}, x_{1}<y_{1}$. Если найдется число $z$, $x_{1}<z \leqslant y_{1}$, такое, что $h(z) \leqslant h\left(x_{1}\right)$, то $x_{1} \in B$. Тогда положим $f(x, y)=y$. Иначе следует положить $f(x, y)=x$, так как из (3) вытекает, что если $x \in B$, то, тем более, $y \in B$. Считаем также, что $f(x, y)=f(y, x)$ при $y_{1} \leqslant x_{1}$. 
Если в случае $x=2 x_{1}+1, y=2 y_{1}+1$ найдется число $z, x_{1}<z \leqslant y_{1}$, такое, что $h(z) \leqslant h\left(x_{1}\right)$, то положим $f(x, y)=x$, а иначе $f(x, y)=y$, считая, по-прежнему, $f(x, y)=f(y, x)$.

Наконец, пусть $x=2 x_{1}, y=2 y_{1}+1$. Перечисляем $B$ и если $x_{1}$ вычислится в $B$, то положим $f(x, y)=f(y, x)=y$. Иначе считаем $f(x, y) \uparrow$. Легко проверить, что $A$ посредством частично-рекурсивной функции $f$ оказьвается wsr*-множеством.

Нетрудно показать, что $A$ не является wsr-множеством [2]. Действительно, если $A$ и $f$ удовлетворяют условию (2), то $f(2 x, 2 x+1) \downarrow$ для всех $x$, причем $x \in B \Longleftrightarrow$ $f(2 x, 2 x+1)=2 x$, что противоречит нерекурсивности $B$. Предположим теперь, что для $A$ нашлась общерекурсивная функция $f$ такая, что выполнено условие (1) и

$R=\left\{\left\langle x_{1}, y_{1}\right\rangle: y_{1}<x \wedge(\forall z)\left(y_{1}<z \leqslant x_{1} \Longrightarrow h\left(y_{1}\right)<h(z)\right)\right\}, \quad x=2 x_{1}, \quad y=2 y_{1}+1$.

Пусть $\left\langle x_{1}, y_{1}\right\rangle \in R$ и $f(x, y)=x$. Тогда

$$
\left(x_{1} \in B \Longrightarrow x \in A \Longrightarrow y \in A \Longrightarrow y_{1} \notin B\right) \wedge\left(x_{1} \notin B \Longrightarrow y_{1} \notin B\right)
$$

Итак, в обоих случаях $y_{1} \notin B$. Но $\mathbb{N} \backslash B$ - иммунное множество, а множество

$$
\left\{y_{1}:\left(\exists x_{1}\right)\left(\left\langle x_{1}, y_{1}\right\rangle \in R \wedge f(x, y)=x\right)\right\}
$$

является его рекурсивно-перечислимым подмножеством и, следовательно, конечно. Поэтому без потери общности можно считать, что $\left\langle x_{1}, y_{1}\right\rangle \in R \Longrightarrow f(x, y) \neq x$.

Пусть теперь $\left\langle x_{1}, y_{1}\right\rangle \in R, y_{1} \notin B$, т.е. $y \in A$. Если $x_{1} \notin B$, то $x \notin A$, поэтому $f(x, y) \neq y$. Но $f(x, y) \neq x$. Значит, $f(x, y)=\bar{a}$. Если же $x_{1} \in B$, то $x \in A$, поэтому $f(x, y)=y$. Это позволяет восстанавливать прямой пересчет $\left\{b_{i}\right\}_{i} \geqslant 0$ дополнения $B$. Действительно,

$$
b_{i+1}=\min \left\{x:\left\langle x, b_{i}\right\rangle \in R \wedge f\left(2 x, 2 b_{i}+1\right)=\bar{a}\right\} .
$$

Пришли к противоречию с нерекурсивностью $B$.

Заметим, что дополнение множества $A$, построенного в теореме 1 , также является wsr*-множеством, потому что оно рекурсивно изоморфно $A$. Отметим еще один результат из [2]: существует wsr-множество такое, что ни оно само, ни его дополнение не являются sre-множествами. В связи с этим представляет интерес

Теорема 2. Если $A$ и $\bar{A}$ - почти полурекурсивные множсества, то $A$ - полурекурсивное множество.

ДоКАЗАТЕЛЬСТво. Пусть $A$ и $\bar{A}$ - почти полурекурсивные множества посредством общерекурсивных функций $f_{1}$ и $f_{0}$ соответственно, $a \in A, \bar{a} \in \bar{A}$. Будем писать $(s, t)$, если $f_{1}(x, y)=s, f_{0}(x, y)=t$, и определять двухместную общерекурсивную функцию $f$, говоря, что ее значение $f(x, y) \in\{x, y\}$ определено правильно, если выполнено условие (1). Легко проверить истинность следующих восьми импликаций, позволяющих 
правильно определить значение:

$$
\begin{array}{ll}
(\bar{a}, x) \Longrightarrow x \in A, & f(x, y)=y ; \\
(\bar{a}, y) \Longrightarrow y \in A, & f(x, y)=x ; \\
(x, a) \Longrightarrow y \in A, & f(x, y)=x ; \\
(y, a) \Longrightarrow x \in A, & f(x, y)=y ; \\
(x, x) \Longrightarrow(x \in A \Longleftrightarrow y \in A), & f(x, y)=x ; \\
(y, y) \Longrightarrow(x \in A \Longleftrightarrow y \in A), & f(x, y)=x ; \\
(x, y) \Longrightarrow(x \in A \Longrightarrow y \in A), & f(x, y)=x ; \\
(y, x) \Longrightarrow(y \in A \Longrightarrow x \in A), & f(x, y)=y .
\end{array}
$$

Однако, в случае $(\bar{a}, a)$ имеем $x \in A \Longleftrightarrow y \in \bar{A}$ и сразу правильно определить значение $f(x, y)$ затруднительно. Но очевидно, что множество $R$ таких пар является рекурсивным. Пусть $(u, v) \in R$, а $(s, t)$ означает, что $f_{1}(x, u)=s$ и $f_{0}(x, v)=t$. Как и вьше, получаем

$$
\begin{aligned}
(x, v) & \Longrightarrow v \notin A, & f(u, v)=v ; \\
(u, x) & \Longrightarrow u \notin A, & f(u, v)=u ; \\
(u, v) & \Longrightarrow x \in A, & f(x, y)=y ; \\
(x, x) \vee(\bar{a}, \bar{a}) & \Longrightarrow x \notin A, & f(x, y)=x .
\end{aligned}
$$

Далее, имеем

$$
(\bar{a}, v) \Longrightarrow(x \in A \Longleftrightarrow v \in A), \quad(u, \bar{a}) \Longrightarrow(x \in A \Longleftrightarrow u \in A) .
$$

В этом случае отождествляем пару $(x, y)$ с $(v, u)$ и $(u, v)$ соответственно. Наконец, имеем

$$
(x, \bar{a}) \Longrightarrow(x \in A \Longrightarrow u \in A), \quad(\bar{a}, x) \Longrightarrow(x \in A \Longrightarrow v \in A) .
$$

Рассмотрим аналогичные импликации с заменой $x$ на $y$. Опять, либо правильно будут определены значения $f(x, y)$ или $f(u, v)$, либо $(x, y)$ будет отождествлена с $(v, u)$ или $(u, v)$, либо

$$
(y, \bar{a}) \Longrightarrow(y \in A \Longrightarrow u \in A), \quad(\bar{a}, y) \Longrightarrow(y \in A \Longrightarrow v \in A) .
$$

Анализируя последние четыре импликации, получаем

$$
\begin{array}{ll}
(x, \bar{a}) \wedge(y, \bar{a}) \Longrightarrow u \in A, & f(u, v)=v ; \\
(\bar{a}, x) \wedge(\bar{a}, y) \Longrightarrow v \in A, & f(u, v)=u ; \\
(x, \bar{a}) \wedge(\bar{a}, y) \Longrightarrow(x \in A \Longleftrightarrow u \in A) ; & \\
(\bar{a}, x) \wedge(y, \bar{a}) \Longrightarrow(x \in A \Longleftrightarrow v \in A) . &
\end{array}
$$

В последних двух случаях отождествляем пару $(x, y)$ с $(u, v)$ и $(v, u)$ соответственно.

Итак, фиксируя $(x, y)$ и перебирая $(u, v) \in R$, можно найти пару $(u, v)$, позволяющую правильно определить $f(x, y)$. Иначе пара $(x, y)$ будет отождествлена с $(u, v)$ или $(v, u)$, либо правильно будет определено значение $f(u, v)$. Тогда в зависимости от того, принадлежит $x$ к $A$ или нет, можно правильно определить значение $f(x, y)$ и, тем самым, $f(u, v)$ для всех пар, эквивалентных $(x, y)$. Можно считать $f(x, y)=f(y, x)$ и $f(x, x)=x$. Тогда $A$ посредством общерекурсивной функции $f$ окажется полурекурсивным множеством.

Следующее предложение обобщает один результат из [1]. 
ПРЕДЛОЖЕНИЕ 2. Простое не әиперпростое множество $A$ не может быть почти полурекурсивным.

ДокАЗАТЕЛЬСтво. Предположим, что $A$ вместе с общерекурсивной функцией $f$ удовлетворяют условию (1). Так как $\bar{A}$ не гипериммунно, то существует общерекурсивная функция $g$ такая, что

$$
(\forall x)(\forall y)\left(x \neq y \Longrightarrow D_{g(x)} \cap D_{g(y)}=\varnothing\right), \quad(\forall x)\left(D_{g(x)} \cap \bar{A} \neq \varnothing\right)
$$

где $D_{n}-$ конечное множество с каноническим номером $n$.

Случай 1. Множество $R$ бесконечно, где

$$
\langle x, y\rangle \in R \Longleftrightarrow(\exists n)\left(x, y \in D_{g(n)} \wedge f(x, y)=\bar{a}\right)
$$

Ясно, что $R$ - рекурсивно-перечислимое множество. Если множество

$$
B=\{\langle x, y\rangle:\langle x, y\rangle \in R \wedge(x \in A \vee y \in A)\}
$$

бесконечно, то множество

$$
C=\{x:(\exists y)((\langle x, y\rangle \in R \wedge y \in A) \vee(\langle y, x\rangle \in R \wedge y \in A))\}
$$

является бесконечным рекурсивно-перечислимым подмножеством $\bar{A}$. Если же множество $B$ конечно, то множество

$$
C^{\prime}=\{x:(\exists y)(\langle x, y\rangle \in R \backslash B)\}
$$

является бесконечным рекурсивно-перечислимым подмножеством $\bar{A}$. Это противоречит иммунности $\bar{A}$.

Случай 2. Множество $R$ конечно (можно считать $R=\varnothing$ ). Для каждого $x$ восстанавливаем $D_{g(x)}=\left\{y_{1}, y_{2}, \ldots, y_{m(x)}\right\}$, где $y_{i}$ также зависят от $x$. Затем вычисляем

$$
d_{2}=f\left(y_{1}, y_{2}\right), \quad d_{3}=f\left(d_{2}, y_{3}\right), \ldots, \quad d_{m(x)}=f\left(d_{m(x)-1}, y_{m(x)}\right)
$$

Из (4) имеем $x \neq y \Longrightarrow d_{m(x)} \neq d_{m(y)} \wedge d_{m(x)} \in \bar{A}$. Следовательно, множество $\left\{d_{m(i)}:\right.$ $i \geqslant 0\}$ опять оказывается бесконечным рекурсивно-перечислимым подмножеством $\bar{A}$.

Ясно, что почти полурекурсивное множество с рекурсивно-перечислимым дополнением является полурекурсивным.

ПРЕДЛОЖЕНИЕ 3. Рекурсивно-перечислимое почти полурекурсивное множество является полурекурсивным. 
ДокАЗАтЕльство. Пусть рекурсивно-перечислимое множество $A$ вместе с общерекурсивной функцией $f$ удовлетворяют условию (1) и

$$
B_{0}=\{x:(\exists y)(y \in A \wedge f(x, y)=\bar{a})\}, \quad B_{n+1}=\left\{x:(\exists y)\left(y \in B_{n} \wedge f(x, y)=x\right)\right\} .
$$

Ясно, что $B=\bigcup_{n \geqslant 0} B_{n}$ является рекурсивно-перечислимым подмножеством $\bar{A}$ и если $\bar{A}=B$, то $A$ - рекурсивное множество. Иначе пусть $b \in \bar{A} \backslash B$ и $R=\{x: f(b, x) \neq b\}$. Тогда $R$ - рекурсивное множество, причем $B \subseteq R \subseteq \bar{A}_{\text {и }}$

$$
\{x, y\} \subseteq \bar{R} \wedge f(x, y)=\bar{a} \Longrightarrow y \notin A .
$$

Положим по определению

$$
g(x, y)= \begin{cases}f(x, y), & \text { если } f(x, y) \in\{x, y\} \wedge x \notin R, \\ x, & \text { если } x \in R, \\ y, & \text { если } x \notin R \wedge f(x, y)=\bar{a} .\end{cases}
$$

Легко проверить, что $A$ посредством общерекурсивной функции $g$ является полурекурсивным множеством.

Бесконечное множество $A \subseteq \mathbb{N}$ называется слабо иммунным [2], если для любого рекурсивно-перечислимого подмножества $B \subseteq A$ найдется рекурсивное множество $R$ такое, что $B \subseteq R \subseteq A$.

ПРЕДЛОЖЕНИЕ 4. Если $A$ - почти полурекурсивное множество, не являющееся рекурсивно-перечислимым, то $A$ - слабо иммунное множсество.

ДокАЗАтЕльство. Пусть $A$ вместе с общерекурсивной функцией $f$ удовлетворяют условию (1), $B$ - рекурсивно-перечислимое подмножество $A, C_{0}=B$ и

$$
C_{i+1}=\left\{x:\left(\exists y \in C_{i}\right)(f(x, y)=y)\right\}, \quad C=\bigcup_{i \geqslant 0} C_{i} .
$$

Так как можно считать, что $f(x, x)=x$ для всех $x \in \mathbb{N}$, то $C_{0} \subseteq C_{1} \subseteq \cdots \subseteq C \subseteq A$. Однако, $C$ - рекурсивно-перечислимое множество, поэтому $C \neq A$. Если $c \in A \backslash C$, а $R=\{x: f(c, x)=c\}$, то $R$ - рекурсивное множество и $R \subseteq A$. Если же $x \in C$ и $f(c, x)=x$, то $x \in C_{i}$ для некоторого $i$. Тогда $c \in C_{i+1} \subseteq C$, что противоречит выбору $c$. Поэтому $x \in C$ влечет $f(c, x)=c$, т.е. $x \in R$. Итак, $B \subseteq C \subseteq R$.

\section{СПИСОК ЦИТИРОВАННОЙ ЛИТЕРАТУРЫ}

[1] Jockusch C. G. Semirecursive sets and positive reducibility // Trans. Amer. Math. Soc. 1968. V. 131. P. 420-436.

[2] Jockusch C. G., Owings J. C. Weakly semirecursive sets and r.e. orderings // J. Symbolic Logic. 1990. V. 55. P. 637-644.

[3] Kummer M., Stephan F. Weakly semirecursive sets and r.e. orderings // Ann. Pure Appl. Logic. 1993. V. 60. P. 133-150.

[4] Розинас М. Г., Солон Б.Я. Слабо полурекурсивные множества // Изв. вузов. Матем. 1979. № 12. C. 48-50.

[5] Yates C. E. M. Recursively enumerable sets and retracing functions // Z. Math. Logik Grundlag. Math. 1962. V. 8. № 4. P. 331-345.

Тюменский государственный университет 